\title{
Effect of Octreotide on the Colonic Motility in Pediatric Patients with Chronic Recalcitrant Constipation.
}

Kalyan Ray Parashette MD MPH ${ }^{1}$

Shamaila Waseem $\mathrm{MD}^{2}$

Debra Horn $\mathrm{RN}^{2}$

Aali Shah $\mathrm{MD}^{3}$

Joseph Croffie MD MPH ${ }^{2}$

${ }^{1}$ Division of Pediatric Gastroenterology, Hepatology and Nutrition, University of New Mexico Children's Hospital, Albuquerque, NM

${ }^{2}$ Division of Pediatric Gastroenterology, Hepatology and Nutrition, Riley Hospital for Children, Indiana University School of Medicine, Indianapolis, IN.

${ }^{3}$ Department of Anesthesia, Riley Hospital for Children, Indiana University School of Medicine, Indianapolis, IN.

\section{Corresponding Author}

Joseph Croffie

Professor of Clinical Pediatrics and Director of Motility Laboratory

Section of Pediatric Gastroenterology, Hepatology and Nutrition

Riley Hospital for Children, Indiana University School of Medicine

705 Riley Hospital Dr., ROC 4210 Indianapolis, IN 46202

Email:jcroffie@iu.edu, Tel: 317-944-3774,Fax: 317-944-8521

This is the author's manuscript of the article published in final edited form as: Parashette, K. R., Waseem, S., Horn, D., Shah, A., \& Croffie, J. (2015). Effect of Octreotide on Colonic Motility in Pediatric Patients With Chronic Recalcitrant Constipation. Journal of pediatric gastroenterology and nutrition, 61(6), 626-629. http://dx.doi.org/10.1097/MPG.0000000000000872 


\begin{abstract}
Objective: To study the effect of octreotide on colonic motility in pediatric patients with recalcitrant chronic constipation/encopresis and other suspected colonic motility disorders.

Methods: This was a non-randomized, single center, open label, prospective study evaluating the effect of a single subcutaneous dose of octreotide on colonic motility Results: Thirteen patients ( 5 male) were enrolled in the study. The age range was 4.616.2 years. Eleven patients $(84 \%)$ had normal colonic manometry and two patients $(16 \%)$ had colonic neuropathy. Motility Index (MI) (mm Hg) for the 15 minutes before and after octreotide infusion was $6.03 \pm 1.26(95 \% \mathrm{CI}, 5.35-6.72)$, and $5.32 \pm 1.66(95 \% \mathrm{CI}, 4.42-$ 6.23) respectively with p-value of 0.08 . MI for the 30 minutes before and after octreotide infusion was $6.89 \pm 1.37(95 \% \mathrm{CI}, 6.14-7.64)$ and $6.71 \pm 1.47(95 \% \mathrm{CI}, 5.91-7.52)$ respectively with p-value of 0.55 . MI for the 45 minutes before and after octreotide infusion was $7.73 \pm 1.32(95 \% \mathrm{CI}, 7.01-8.45)$ and $7.53 \pm 1.38(95 \% \mathrm{CI}, 6.78-8.28)$ respectively with p-value of 0.8 .

Conclusion: Our study showed that administration of octreotide resulted in no significant changes in colonic motility index in pediatric patients with chronic recalcitrant constipation.
\end{abstract}

Key words: Constipation, Octreotide, Colonic manometry, Colonic dysmotility, Motility Index

\title{
What is Known/What is New:
}

- Treatments options for patient with intractable constipation and colonic dysmotility are very limited. 
- Octreotide induces phase III of the migrating motor complex in the small intestine. Octreotide causes no significant changes in colonic motility index in pediatric patients with chronic recalcitrant constipation with normal colonic manometry.

\section{Introduction}

Constipation is a common problem worldwide with an estimated prevalence of $3 \% .{ }^{1}$ In the Western world $10-20 \%$ of adults have constipation. ${ }^{2}$ Constipation is commonly encountered in pediatrics as well, contributing to $3 \%$ of general pediatrician office visits and $25 \%$ of pediatric gastroenterology visits. ${ }^{3}$ Bongers et al studied the long-term prognosis of pediatric patients with constipation; nearly $30 \%$ of patients continued to be symptomatic despite aggressive medical and behavioral therapy, with a mean follow up of 11 years. ${ }^{4}$ These subsets of patients with intractable constipation often need a colonic motility study to determine if their symptoms are due to a colonic dysmotility. ${ }^{5}$ Based on the results of the study, appropriate therapeutic interventions may be recommended. Patients with intractable constipation and normal colonic motility are often offered surgical treatment (MACE/cecostomy) if all medical therapy has failed. ${ }^{6}$ Patients with true dysmotility may need MACE/cecostomy or total or segmental colonic resection depending on severity of colonic dysmotility. There is a need to identify newer medical therapies to treat patients with intractable constipation and colonic dysmotility as treatments options are very limited..$^{5}$

Octreotide is a synthetic octapeptide analog of somatostatin. ${ }^{7}$ It has several

gastrointestinal actions. ${ }^{8-11}$ Octreotide induces phase III of the migrating motor complex in 
the small intestine. ${ }^{12}$ Von der Ohe et al conducted a randomized blind study assessing the regional effects of octreotide on the gastrointestinal tract; the study showed improvement in the colonic motility index and statistically significant phasic pressure activity in the octreotide group compared to placebo. ${ }^{13}$ Soudah et al showed that octreotide stimulated rectosigmoid motility via cholinergic pathways resulting in improved colonic transit. ${ }^{14}$ Scarpignato et al used octreotide in small pulse doses in patients with irritable bowel syndrome and noticed that it accelerated intestinal transit. ${ }^{15}$ Cullen et al used octreotide in postoperative ileus and showed that octreotide has a dose dependent action on colonic motility; used in low doses it acted as a prokinetic agent and resulted in patients having a bowel movement earlier. ${ }^{16}$

We have used octreotide during colonic motility studies in seven patients who had no response to a standard dose of bisacodyl. All seven patients had high amplitude propagated colonic contractions (HAPC) within 5-10 minutes of administration of octreotide and tolerated the medication well. ${ }^{17}$ However, there might have been a confounding factor, given that all patients received bisacodyl prior to octreotide.

The aim of this pilot study was to investigate the effect of octreotide on colonic motility in pediatric patients with recalcitrant chronic constipation/encopresis and other suspected colonic motility disorders.

\section{Materials and Methods}

This was a non-randomized, single center, open label, and prospective study. The study was approved by the institutional review board of Indiana University after obtaining an Investigational New Drug (IND) application from the Food and Drug 
Administration (FDA). This study was registered at clinical trials and trial identification number is NCT01917773.

For inclusion criterion any child between one to eighteen years of age who was undergoing a colonic motility study at Riley Hospital for Children was eligible to participate in the study. The exclusion criteria were: 1) subjects with known or suspected allergy to octreotide, 2) subjects with known prolonged QTc Syndrome or highest risk QTc-Prolonging Agents (including mifepristone), 3) subjects with known history of ventricular arrhythmia, 4) subjects with history of organ transplant taking cyclosporine at the time of the motility study, 5) subjects with history of small bowel transplant, 6) subjects less than one year or older than eighteen years of age, 7) subjects with severe renal impairment, 8) subjects with severe hepatic impairment, 9) subjects taking bromocriptine, insulin, oral hypoglycemic agents, beta blockers, calcium channel blockers, quinidine, terfenadine, pimozide, sildenafil, tadalafil, and any agents to control fluid and electrolyte imbalance.

All eligible patients referred for colonic manometry to the Gastrointestinal Motility Laboratory at Riley Hospital for Children between September 2013 and June 2014 were invited to participate in the study. Informed consent was obtained from parents/legal guardian of each enrolled child and assent was obtained from all patients older than 7 years.

Protocol of Colonic Manometry

Laxatives were discontinued 48 hours before colonic manometry. A standard bowel preparation (oral or nasogastric infusion of polyethylene glycol/electrolyte) was 
prescribed the day before colonoscopy for motility catheter placement. On the day of the motility catheter placement colonoscopy was performed, and a motility catheter was placed under direct visualization. Colonoscopy was performed under standardized general anesthesia protocol consisting of premedication with midazolam and induction and maintenance of anesthesia using sevoflurane. The motility catheter was grasped by a polypectomy snare and was advanced to the most proximal point in the colon that was accessible. A plain abdominal x-ray was ordered within two to three hours after completion of the procedure and again in the middle of the colonic motility study to document the approximate location of the pressure ports on the catheter.

The protocol for manometry was as follows.

1. Fasting motility was recorded for at least 60 minutes.

2. Octreotide $1 \mathrm{mcg} / \mathrm{kg}$, maximum of $50 \mathrm{mcg}$ was administered subcutaneously (one hour after application of EMLA topical cream). Motility was then recorded for 45-60 minutes.

3. Patients were then offered a high-fat, high-energy meal as described elsewhere, and motility was recorded for 60 minutes. ${ }^{18}$

4. Patients then received 1 to 2 doses of bisacodyl $(0.2 \mathrm{mg} / \mathrm{kg}$, maximum of $10 \mathrm{mg})$ through the motility catheter, and motility was recorded. ${ }^{19}$

Data and Statistical Analysis:

Colonic motility was measured using a solid-state catheter. The catheter had 36 sensors spaced $5 \mathrm{~cm}$ apart for the first 15 sensors and $1 \mathrm{~cm}$ apart for the remaining sensors. 
Pressures were transmitted to a transducer and recorded on a personal computer system (Medical Measurement Systems USA, Inc.). Manometry tracings were visually analyzed for the presence of high-amplitude propagating contractions (HAPCs), defined as contractions of at least $60 \mathrm{~mm} \mathrm{Hg}$ in amplitude, 10 seconds in duration, and propagating for at least $30 \mathrm{~cm}$ of the colon. Normal manometry was defined as the presence of at least one HAPC originating in the proximal colon and propagating to the sigmoid colon. ${ }^{20}$ Neuropathy was defined as the persistent presence of a pattern of low- or high-amplitude contractions that were non-propagating (retrograde or simultaneous). Myopathy was defined as complete absence of contractions or persistence of a pattern of contractions with normal propagation but abnormally low amplitude in a non-dilated colon. Motility index (MI) was calculated using the Medical Measurement Systems (MMS) computer program. The MI represents the area under the curve of the pressure tracing for a certain period. ${ }^{21}$ The MI was calculated for each channel. The MIs from all channels were then averaged to give each patient one average MI for the particular period under study. In this study, MI was calculated for the periods of 15,30 , and 45 minutes before and after infusion of octreotide. Motility index is reported as millimeters of mercury ( $\mathrm{mm} \mathrm{Hg}$ ) per 15,30 or 45 minutes.

There are no commonly accepted norms for colonic motility index in children using the solid-state colonic motility catheter technique. Reported MIs and SDs in healthy volunteers from an adult study were used to calculate sample size. ${ }^{22} \mathrm{~A}$ sample size of 13 patients was deemed adequate to detect a $25 \%$ change in MI with $80 \%$ power. The Wilcoxon signed rank test was used to analyze change in MI. Values were considered to be significant if $\mathrm{P}<0.05$. 


\section{Results}

Demographics:

Twenty-four patients were assessed for eligibility to participate in the study. Eight patients were excluded, as they did not meet inclusion criteria. Three patients/parents refused to be part of the study. Thirteen patients were enrolled in the study. The age range was 4.6-16.2 years with a mean age of $9.3 \pm 3.3$ years. Seven patients were male and six patients were female. Twelve patients were white and one patient was black. Indications for colonic manometry included refractory constipation with encopresis (11), constipation without encopresis (1) and constipation post pull-through procedure for Hirschsprung's disease (1). Seventy seven percent of patients had significant comorbidities (Table 1). Eleven patients had undergone anorectal manometry (ARM) and results showed presence of recto-anal inhibitory reflex and normal defecation dynamics (5), presence of RAIR with paradoxical contraction during simulated defecation (3), absent RAIR and normal rectal biopsy (2), and absent RAIR post Hirschsprung's disease (HD) surgery (1) (Table

1). All three patients with positive RAIR with paradoxical contraction during simulated defecation had failed biofeedback therapy. Six patients had undergone rectal biopsy and ganglion cells were present in five patients (one was diagnosed with Hirschsprung's disease).

A motility catheter was placed via colonoscopy in all 13 patients. The catheter was advanced to the cecum in 7 patients, ascending colon in 3 and hepatic flexure in 3. Six patients complained of mild discomfort with administration of subcutaneous 
octreotide but did not require any analgesic, as discomfort was short lived. Three patients complained of mild abdominal cramping. None of the patients developed hypoglycemia.

Qualitative analysis of effect of Octreotide:

Eleven patients had normal manometry. Two patients had colonic neuropathy. Of the 11 healthy patients, none had fasting HAPCs, one had post-octreotide HAPCs, two had postprandial HAPCs, and all had post bisacodyl HAPCs. Octreotide failed to produce any noticeable visual changes in the manometry pattern compared to the fasting period in all but two patients.

Quantitative analysis of effect of Octreotide:

Average MI for all patients was calculated over 15-minutes, 30-minutes and 45minutes before and after administration of octreotide. The MI for the 15 minutes before and after octreotide infusion was $6.03 \pm 1.26 \mathrm{~mm} \mathrm{Hg} / 15 \mathrm{~min}(95 \%$ confidence interval, 5.35-6.72), and $5.32 \pm 1.66 \mathrm{~mm} \mathrm{Hg} / 15 \mathrm{~min}$ (95\% confidence interval, 4.42-6.23) and pvalue was $\mathrm{P}=0.087$. The $\mathrm{MI}$ for the 30 minutes before and after octreotide infusion was $6.89 \pm 1.37 \mathrm{~mm} \mathrm{Hg} / 30 \mathrm{~min}(95 \%$ confidence interval, $6.14-7.64)$ and $6.71 \pm 1.47 \mathrm{~mm}$ $\mathrm{Hg} / 30$ min ( $95 \%$ confidence interval, 5.91-7.52) and $\mathrm{p}$-value was $\mathrm{P}=0.552$. MI for the 45 minutes before and after octreotide infusion was $7.73 \pm 1.32 \mathrm{~mm} \mathrm{Hg} / 45 \mathrm{~min}(95 \%$ confidence interval, $7.01-8.45$ ) and $7.53 \pm 1.38 \mathrm{~mm} \mathrm{Hg} / 45 \mathrm{~min}$ (95\% confidence interval, 6.78-8.28) and $\mathrm{p}$-value was $\mathrm{P}=0.807$. Motility index increased in three, six and seven patients at 15, 30 and 45 minutes after administration of octreotide, respectively (Figure. 1). However, overall motility index decreased post octreotide during each time period. 
Only in one patient MI increased more than 25\% from baseline following administration of octreotide (patient 11).

A sub analysis of 11 patients with normal colonic manometry showed no significant changes in MI 15 minutes, 30 minutes and 45 minutes after octreotide administration. The MI for the 15 minutes before and after octreotide was $5.99 \mathrm{~mm}$ $\mathrm{Hg} / 15 \mathrm{~min}$ and $5.27 \mathrm{~mm} \mathrm{Hg} / 15 \mathrm{~min}$ (p-value 0.075 ) respectively. The MI for the 30 minutes before and after octreotide was $6.84 \mathrm{~mm} \mathrm{Hg} / 30 \mathrm{~min}$ and $6.64 \mathrm{~mm} \mathrm{Hg} / 30 \mathrm{~min}$ (pvalue 0.534$)$ respectively. The MI for the 45 minutes before and after octreotide was 7.66 $\mathrm{mm} \mathrm{Hg} / 45 \mathrm{~min}$ and $7.5 \mathrm{~mm} \mathrm{Hg} / 45 \mathrm{~min}$ (p-value 0.86 ) respectively.

A secondary analysis on all 13 patients post bisacodyl infusion showed no significant changes. The average 15 minutes MI post-bisacodyl was $6.7 \mathrm{~mm} \mathrm{Hg}$ compared to pre-bisacodyl MI of $6.032 \mathrm{~mm} \mathrm{Hg}$ (p-value 0.249). Reanalysis of the 11 patients with normal colonic manometry alone showed significant changes post bisacodyl infusion as expected. The average 15 minutes MI post-bisacodyl was $7.2 \mathrm{~mm} \mathrm{Hg}$ compared to pre-bisacodyl MI of $5.992 \mathrm{~mm} \mathrm{Hg}$ (p-value 0.049).

\section{Discussion}

Octreotide is a synthetic octapeptide analogue of somatostatin. Octreotide is used to treat several pediatric disorders including secretory diarrhea, gastrointestinal hemorrhage, dumping syndrome, gastrointestinal fistula and pseudo obstruction. ${ }^{11}$ Octreotide has been shown to have several actions on the colon including increased phasic pressure activity, and increased transit time. ${ }^{13-16}$ We chose to use the dose of $1 \mathrm{mcg} / \mathrm{kg}$ with a maximum of $50 \mathrm{mcg}$ based on reports in the literature. ${ }^{23} \mathrm{~A}$ similar 1 
$\mathrm{mcg} / \mathrm{kg}$ dose has been used during antroduodenal motility studies in pediatric patients to induce phase III of the migrating motor complex. Di Lorenzo et al reported using this dose in their prospective study of pediatric patients undergoing antroduodenal motility studies without any adverse effects. ${ }^{12}$ Also, octreotide is used for gastrointestinal bleeding where $1 \mathrm{mcg} / \mathrm{kg}$ is given as a bolus intravenous dose, followed by $1 \mathrm{mcg} / \mathrm{kg} / \mathrm{hr}$ for 48 hours. ${ }^{24}$ We used sevoflurane anesthesia protocol as we have shown previously that anesthesia protocol does not alter colonic motility study findings. ${ }^{25}$

In our study octreotide was overall well tolerated. Six patients had mild discomfort with administration of subcutaneous octreotide. Three patients had mild abdominal discomfort.

In this study, the effects of a single subcutaneous dose of octreotide on colonic motility was evaluated in pediatric patients with chronic recalcitrant constipation. The study shows that octreotide did not increase colonic MI. In fact in the first 15 minutes post octreotide the MI decreased. Octreotide induced HAPC's in only one patient. Based on this observation we conclude that octreotide is unlikely to benefit patients with chronic intractable or recalcitrant constipation.

We have used octreotide previously in patients who had no HAPC's after bisacodyl infusion during a colonic motility study. Octreotide induced HAPC's within 5-10 minutes in most of these patients. ${ }^{17}$ When we compare this study result to our previous experience, it appears that octreotide may augment bisacodyl's effects but is not able to induce HAPC's on its own.

Our study has a few limitations. We did not have a healthy control group; however, it will be difficult to enroll healthy controls and expose them to an invasive and 
expensive procedure. The study was an open-label design and not a randomized double blind control study; however, the risk of bias is minimal as we are studying an objective data, i.e., colonic MI, which is computer generated. As we had only two patients with colonic neuropathy, it is difficult to conclude effect of octreotide in colonic neuropathy patients.

In conclusion our study shows that administration of octreotide resulted in no significant changes in colonic motility index in pediatric patients with chronic recalcitrant constipation with normal colonic manometry study. Additional studied are needed to assess response of octreotide in patients with colonic neuropathy and myopathy. We previously have shown that octreotide induced HAPC's in patients who had no response to a standard dose of bisacodyl. This observation suggests that octreotide likely enhances the effect of bisacodyl. However further research is needed to confirm this hypothesis.

\section{References:}

1] van den Berg MM, Benninga MA, Di Lorenzo C. Epidemiology of childhood constipation: a systematic review. Am J Gastroenterol 2006;101:2401-9.

2] Talley NJ, O'Keefe EA, Zinsmeister AR, et al. Prevalence of gastrointestinal symptoms in the elderly: a population-based study. Gastroenterology 1992; 102: 895901.

3] Molnar D, Taitz LS, Urwin OM, et al. Anorectal manometry results in defecation disorders. Archives of disease in childhood. Apr 1983;58(4):257-261. 
4] Bongers ME, Benninga MA. Long-term follow-up and course of life in children with constipation. Journal of pediatric gastroenterology and nutrition. Dec 2011;53 Suppl 2:S55-56.

5] Tabbers MM, DiLorenzo C, Berger MY, et al. Evaluation and treatment of functional constipation in infants and children: evidence-based recommendations from ESPGHAN and NASPGHAN. Journal of pediatric gastroenterology and nutrition. Feb $2014 ; 58(2): 258-274$.

6] Youssef NN, Pensabene L, Barksdale E, Jr, et al C. Is there a role for surgery beyond colonic aganglionosis and anorectal malformations in children with intractable constipation? Journal of pediatric surgery. Jan 2004;39(1):73-77.

7] Katz MD, Erstad BL. Octreotide, a new somatostatin analogue. Clinical pharmacy. Apr $1989 ; 8(4): 255-273$.

8] Benson AB, 3rd, Ajani JA, Catalano RB, et al. Recommended guidelines for the treatment of cancer treatment-induced diarrhea. Journal of clinical oncology : official journal of the American Society of Clinical Oncology. Jul 15 2004;22(14):2918-2926.

9] Corley DA, Cello JP, Adkisson W, et al. Octreotide for acute esophageal variceal bleeding: a meta-analysis. Gastroenterology. Mar 2001;120(4):946-954.

10] Couper RT, Berzen A, Berall G, et al. Clinical response to the long acting somatostatin analogue SMS 201-995 in a child with congenital microvillus atrophy. Gut. Jul 1989;30(7):1020-102.

11] Heikenen JB, Pohl JF, Werlin SL, et al. Octreotide in pediatric patients. Journal of pediatric gastroenterology and nutrition. Nov 2002;35(5):600-609. 
12] Di Lorenzo C, Lucanto C, Flores AF, et al. Effect of octreotide on gastrointestinal motility in children with functional gastrointestinal symptoms. Journal of pediatric gastroenterology and nutrition. Nov 1998;27(5):508-512.

13] von der Ohe MR, Camilleri M, Thomforde GM, et al. Differential regional effects of octreotide on human gastrointestinal motor function. Gut. May 1995;36(5):743-748.

14] H Soudah, W. H., C Owyang. Somatostatin analog stimulates rectosigmoid motility and promotes colonic transit in humans via a cholinergic pathway. Gastroenterology. 1991;100:A497.

15] Scarpignato C, Pelosini I. Management of irritable bowel syndrome: novel approaches to the pharmacology of gut motility. Canadian journal of gastroenterology Journal canadien de gastroenterologie. Mar 1999;13 Suppl A:50A-65A.

16] Cullen JJ, Eagon JC, Dozois EJ, et al. Treatment of acute postoperative ileus with octreotide. American journal of surgery. Jan 1993;165(1):113-119; discussion 119-120.

17] Croffie JM, Horn D, Corkins MR, et al. Octreotide induces high amplitude propagated colonic contractions (HAPCs). J Pediatr Gastroenterol Nutr 2001;33:405 A. 18] Di Lorenzo C, Hillemeier C, Hyman P, et al. Manometry studies in children: minimum standards for procedures. Neurogastroenterology and motility : the official journal of the European Gastrointestinal Motility Society. Aug 2002;14(4):411-420.

19] De Schryver AM, Samsom M, Smout AI. Effects of a meal and bisacodyl on colonic motility in healthy volunteers and patients with slow-transit constipation. Digestive diseases and sciences. Jul 2003;48(7):1206-1212. 
20] van den Berg MM, Hogan M, Caniano DA, et al. Colonic manometry as predictor of cecostomy success in children with defecation disorders. Journal of pediatric surgery. Apr 2006;41(4):730-736; discussion 730-736.

21] Camilleri M, Bharucha AE, di Lorenzo C, et al. American Neurogastroenterology and Motility Society consensus statement on intraluminal measurement of gastrointestinal and colonic motility in clinical practice. Neurogastroenterology and motility : the official journal of the European Gastrointestinal Motility Society. Dec 2008;20(12):1269-1282.

22] Rao SS, Kavelock R, Beaty J, Ackerson K, et al. Effects of fat and carbohydrate meals on colonic motor response. Gut. Feb 2000;46(2):205-211.

23] Haruma K, Wiste JA, Camilleri M. Effect of octreotide on gastrointestinal pressure profiles in health and in functional and organic gastrointestinal disorders. Gut. Aug 1994;35(8):1064-1069.

24] Siafakas C, Fox VL, Nurko S. Use of octreotide for the treatment of severe gastrointestinal bleeding in children. Journal of pediatric gastroenterology and nutrition. Mar 1998;26(3):356-359.

25] Ammoury RF, Emhardt JD, Aitchison WB, et al. Can colonic manometry studies be done on the day of colonic motility catheter placement? Journal of pediatric gastroenterology and nutrition. 2012 Sep;55(3):278-82. 